\title{
Upcoming Events in Pediatric Cardiology
}

(c) Springer Science+Business Media New York 2017

October 15-19, 2017

American Academy of Pediatrics National Conference Chicago, IL, USA

http://www.aapexperience.org/

November 11-15, 2017

Scientific Session, American Heart Association

Anaheim, CA, USA

http://professional.heart.org

January 27-31, 2018

Annual Meeting of the Society of Thoracic Surgery

Fort Lauderdale, FL, USA

http://www.sts.org

September 5-8, 2018

Pediatric Interventional Cardiology Symposium-AICS

Las Vegas, NV, USA

http://www.picsymposium.com/
April 10-12, 2018

American College of Cardiology, Annual Scientific Session Orlando, FL, USA

http://www.acc.org

April 28-May 2, 2018

American Association of Thoracic Surgery, Annual Meeting

San Diego, CA, USA

http://www.aats.org 